\title{
Colon cancer cells secrete exosomes to promote self-proliferation by shortening mitosis duration and activation of STAT3 in a hypoxic environment
}

\author{
Ruixue Ren, Hua Sun, Cui Ma, Jiatao Liu and Hua Wang* ${ }^{*}$
}

\begin{abstract}
Colon-cancer-cell-derived exosomes (CDEs) are emerging mediators of tumorigenesis and serve as messengers of intercellular communication; however, whether the CDEs affect the proliferation of colon cancer cells themselves remains unknown. In the current study, the CDEs isolated from human colon cancer cell line SW480 and HCT116 showed a size range of 60-150 nm, typical bilayer-encapsulated vesicles, and expressed the exosomal markers CD81 and CD63. Incubation of SW480 cells with CDEs labelled with PKH67 fluorescent markers revealed that SW480 cells were able to absorb CDEs, which were mostly distributed around the nucleus. Hypoxic conditions promoted colon cancer cells to release a greater number of CDEs than normoxic conditions. MTT cell proliferation assay demonstrated CDEs promoted the proliferation of colon cancer cells in a time- and dose-dependent manner. Mechanistically, CDEs promoted colon cancer cell growth mainly through shortening mitosis duration. Meanwhile, the levels of phosphorylated STAT3 in colon cancer cells was up-regulated with the treatment of CDEs derived from hypoxic tumor cells. Our data suggests that colon cancer cells are able to promote self-growth through the secretion of exosomes, especially under hypoxic conditions, which shortens mitosis duration and activates STAT3.
\end{abstract}

Keywords: Exosomes, Hypoxia, Cell proliferation, Mitosis, STAT3

\section{Introduction}

As the third most common cancer in the world, colorectal cancer (CRC), especially metastatic CRC, has received great attention in recent years due to its high morbidity and mortality [1]. Similarly, the incidence and mortality of CRC in China have also increased significantly [2]. The occurrence and development of CRC may be related to many risk factors, such as smoking, obesity and red meat consumption, and the recurrence and metastasis of CRC is the main cause of death [3]. Despite the great progress in the treatment of CRC including surgery and combined radiation therapy and chemotherapy, the 5-year survival rate of CRC is still poor and the overall prognosis of CRC

*Correspondence: wanghua@ahmu.edu.cn

Department of Oncology, The First Affiliated Hospital of Anhui Medical

University, 218 Jixi Road, Hefei 230022, Anhui, China is still not optimistic [4]. Therefore, the molecular mechanisms for exploring its occurrence and development needed to be explored urgently.

Small extracellular vesicles (also known as exosomes) can be released by tumor cells to promote tumor progression, playing an important role in communicating between tumor cells and their microenvironment [5]. Exosomes are membrane-enclosed vesicles approximately $30-150 \mathrm{~nm}$ in diameter and are widely distributed in various body fluids. Colon cell-derived exosomes (CDEs) have been shown to modulate local and systemic tumor microenvironment by transferring bioactive factors, such as nucleic acids and proteins [6]. However, the specific composition of exosomes depends on their parental cell type, as well as environmental factors, such as hypoxia. 
Tumor hypoxia is a common phenomenon in solid tumors and has been widely considered as one of the most fundamental tumor microenvironment stresses for solid tumors [7]. Tumor hypoxia not only causes the problem affecting therapeutic efforts but may also acts as a selective pressure promoting tumor aggressiveness [7, 8]. In a hypoxic microenvironment, tumor cells reshape their microenvironment to sustain survival and rapid growth [7,9], this results in the continuous selection of CRCs that have acquired abilities to withstand these harsh hypoxic conditions and adapt to a hypoxic microenvironment, thereby enhancing tumor proliferation [5]. In addition, hypoxia has been shown to increase exosome secretion and cancer invasion in many types of tumors. Hypoxia-inducible factors promote the formation of microvesicles that stimulate tumor cancer invasion and metastasis [7, 10]. Exosomes secreted under hypoxia enhance invasiveness of prostate cancer cells by targeting adherens junction molecules [11]. Hypoxia-induced exosomes induce a more aggressive and chemoresistant ovarian cancer phenotype [12]. All these studies demonstrate an important role of hypoxia related exosomes in exacerbating malignant progression of tumors.

The dysregulation of normal cell cycle control is a hallmark of human cancer. A variety of tumor cell-derived exosomes can transmit miRNA and mRNA to regulate cell cycle [13-15]. For example, exosomes derived from human primed mesenchymal stem cells induce mitosis of themselves [16]. At present, mitosis has attracted widespread attention as a chemotherapeutic target, and numerous strategies for targeting the cancer cell cycle have been proposed. However, whether the colon cancer-derived exosomes (CDEs) regulate self-proliferation by altering colon cancer cell cycle remains unknown. In the current study, we examined the effects of the CDEs isolated from human colon cancer cell line SW480 and HCT116 on proliferation of these cell lines and explored underlying mechanisms were also explored.

\section{Materials and methods}

\section{Cell lines and culture}

The cell lines SW480 and HCT were purchased from the Cell Bank of the Chinese Academy of Sciences (Shanghai, China). These cell lines were immediately expanded and frozen so that they could be restarted every 10 generation from a frozen vial of the same batch of cells. The SW480 and HCT cells were cultured in DMEM supplemented with $10 \%$ fetal bovine serum (Gibco, Grand Island, NY, USA) and penicillin/streptomycin $(100 \mathrm{IU} / \mathrm{ml}$ and $100 \mathrm{mg} / \mathrm{ml}$, respectively; Beyotime Biotechnology, Jiangsu, China). Cells were maintained at $37{ }^{\circ} \mathrm{C}$ in a $5 \%$ $\mathrm{CO}_{2}$ atmosphere. These cells were cultured under normoxic condition (normoxic group) and hypoxia model condition (co-cultured with $\mathrm{CoCl}_{2}$ to mimic hypoxia condition). The cells that were cultured with $10-\mathrm{cm}$ dish was used as exosomes collection and the cells were cultured with 96-well plates was used as a subsequent cell proliferation assay.

\section{Antibodies}

Primary antibodies used in this study included rabbit anti-CD63 (System Biosciences, USA), rabbit anti-CD81 (System Biosciences, USA), rabbit anti-Calnexin (Cell Signaling, China), mouse anti- $\beta$-tubulin (Sigma), rabbit anti-phospho-Histone H3 (Ser10) (Milipore Sigma), rabbit anti-Phospho-Stat3 (Tyr705), rabbit anti-STAT3, rabbit anti-Cyclin D1, rabbit anti-Hes1 (Cell Signaling, China). Secondary antibodies used in this study were goat anti-rabbit or mouse HRP, donkey anti-rabbit or mouse Cy5, goat anti-rabbit or mouse Alexa 594, goat anti-human FITC or goat anti-rabbit or mouse Alexa 488 (Jackson Immuno Research, West grove, PA, USA).

\section{Purification of exosomes from cell culture supernatants}

SW480 cells were plated on $10-\mathrm{cm}$ dishes at a density of $1 \times 10^{6}$ cells per dish in the culture media described above. After $48 \mathrm{~h}$, culture media were discarded, cells were washed three times with PBS, and $5 \mathrm{ml}$ of serumfree culture medium was added to each dish. After $48 \mathrm{~h}$ or $72 \mathrm{~h}$ ' cell culture in normoxic condition (Exo-normoxic) and hypoxia condition (Exo-hypoxic) (cells were incubated in the presence of $100 \mu \mathrm{M} \mathrm{CoCl}_{2}$ to mimic the hypoxia condition for $24 \mathrm{~h}$ ), then the cell culture media were collected and filtered using a $0.22-\mu \mathrm{m}$ filter and collected in new tubes. The supernatants were centrifuged at $3000 \times g$ for 15 min to remove cells and cell debris. Then, supernatants were transferred to sterile vessels and $1.2 \mathrm{ml}$ of Exo Quick-TC was added. And mixed well by inverting or flicking the tube. After refrigerating overnight (usually $18 \mathrm{~h}$ ), the Exo Quick-TC/biofluid mixture was centrifuged at $4000 \times g$ for 35 min to collect exosomes. The exosomal pellets were resuspended in $120 \mu \mathrm{l}$ using sterile $1 \times$ PBS.

\section{PKH67 labeling of exosomes and exosomes uptake into recipient cells}

SW480-derived exosomes were collected from $100 \mathrm{ml}$ of culture medium (20 10-cm culture dishes were used) as described above. The $5 \mu \mathrm{g}$ exosomes for PKH67 labeling. Exosomes were labeled using PKH67 Fluorescent Cell Linker kits (Sigma-Aldrich, St. Louis, MO) according to the manufacturer's instructions, with minor modifications.

To examine the uptake of exosomes into recipient CRC cells, DMEM containing either PKH67-labeled exosomal solution or control solution was added to each well. Cells 
were cultured for $24 \mathrm{~h}$ at $37{ }^{\circ} \mathrm{C}$ in a normal atmosphere with $5 \% \mathrm{CO}_{2}$. The slides were washed three times with D-PBS(-) and fixed with $3.7 \%$ formaldehyde solution at room temperature for $10 \mathrm{~min}$. Slides were then washed three times in D-PBS(-). After the staining of nuclei using a Pro Long Gold Antifade Reagent with 4',6-diamidino-2-phenylindole (DAPI; Life Technologies), the slides were covered with coverslips and visualized under a confocal laser scanning microscope (LSM710; Carl Zeiss, Oberkochen, Germany).

\section{Immunofluorescence}

Cells plated on coverslips were pre-extracted with $0.2 \%$ Triton X-100 in PHEM for $45 \mathrm{~s}$ before fixation with $4 \%$ paraformaldehyde in PBS. After staining, experiments for CRC cells were fixed directly in $4 \%$ paraformaldehyde before extraction. Then, cells were blocked with $1 \%$ bovine serum albumin in TBST for $30 \mathrm{~min}$, incubated with primary antibodies for $2 \mathrm{~h}$ at room temperature, washed with TBST three times and incubated with secondary antibodies for an additional $1 \mathrm{~h}$ at room temperature. DNA was stained with 4,6-diamidino2-phenylindole for 2-3 min. Images were acquired using a DeltaVision microscope (GE Healthcare, Buckinghamshire, UK).

\section{Western blotting}

The exosomes isolated by Exoquick ${ }^{\mathrm{TM}}$ precipitation or colon cancer cells were lysed with RIPA buffer (Sigma, USA). The protein concentration of lysates using the Bradford Assay Kit (Abcam, USA) with Thermo Scientific $^{\mathrm{TM}}$ NanoDrop ${ }^{\mathrm{TM}}$ One (USA). Samples were subjected to SDS-PAGE on $12 \%$ tris-glycine gels and blotted onto nitrocellulose membranes. Membranes were probed with specific primary antibodies over-night at $4{ }^{\circ} \mathrm{C}$ followed secondary antibody for $1 \mathrm{~h}$ at room temperature and visualized by the ECL detection system.

\section{Live-cell imaging}

For live-cell imaging, cells on coverslips were mounted in Rose chambers and maintained at $37^{\circ} \mathrm{C}$ in phenol-free L-15 medium (Invitrogen) with 10\% fetal bovine serum. Time-lapse images were acquired at $3 \mathrm{~min}$ intervals with a $100 \times 1.4$ NA PlanApo objective lens mounted on an Eclipse Ti microscope (Nikon, Tokyo, Japan). Z-stacks were collected at $1-\mu \mathrm{m}$ steps.

\section{Electron microscopy}

The purified CDEs were treated with RNase A to degrade any non-CDEs RNA. SW480 Exo-normoxic and Exo-hypoxic were analyzed for the size of particles and morphology by transmission electron microscope (TEM). Exosomes were suspended in glutaraldehyde, and $\sim 3-5 \mu \mathrm{l}$ of exosomes were applied to 400 mesh copper grids (formvar/carbon coated, glow-discharged) for $5 \mathrm{~min}$, followed by negative staining with $2 \%$ uranyl acetate for $2 \mathrm{~min}$. Grids were briefly washed in water, allowed to dry and viewed using FEI Technai Transmission electron microscope.

\section{MTT assay}

SW480 and HCT116 cell lines $\left(3-5 \times 10^{5} / \mathrm{ml}\right)$ were cultured in 96-well plates and then treated with the required reagents in DMEM with 5\% FBS. Then, the samples were exposed to hypoxia (use $\mathrm{CoCl}_{2}$ at the final concentration of $100 \mu \mathrm{M}$ in our cell culture media to induce hypoxia). Add the $\mathrm{CoCl}_{2}$ containing media to our cells and incubate the cultures for $24 \mathrm{~h}$ in a conventional incubator $\left(37^{\circ} \mathrm{C} ; 5 \% \mathrm{CO}_{2}\right)$ for $24 \mathrm{~h}$. At the end of the incubation at $37{ }^{\circ} \mathrm{C}$, the PAECs were incubated for another $4 \mathrm{~h}$ in a medium containing $0.5 \%$ 3-[4,5-dimethylthiazol-2-yl]2,5-diphenyl-tetrazolium bromide (MTT). The reaction was terminated by the addition of DMSO to the medium. The absorbance at $540 \mathrm{~nm}$ was measured using a spectrophotometer.

\section{Statistical analysis}

Data are expressed as the mean \pm SEM and were analyzed using GraphPad Prism software (v. 5.0a; GraphPad Software, La Jolla, CA). To compare values obtained from two groups, the Student $t$ test was performed. P values of $<0.05$ were considered significant. Ted intensities of the full nucleus were measured by ImageJ. Data were from three or more independent experiments.

\section{Results}

The secretion of CDEs is increased when colon cancer cells are exposed to a hypoxic environment

As shown in Fig. 1a, TEM revealed the "cup-shaped" vesicles of CDEs, which is consistent with previous reports [17]. TEM results showed that the average size of Exonormoxic $(150 \mathrm{~nm} \pm 5)$ was larger than Exo-hypoxic $(60 \mathrm{~nm} \pm 5)$. We also quantified the number of CDEs (4 samples were included in each group). Furthermore, we also characterized four exosomal protein markers of exosomes derived from two different CRC cell lines (SW480 and HCT116 cells) by western blotting analysis. The membrane protein calnexin is not expressed in CDEs, indicating that a high purity of CDEs. The CDEs marker proteins CD63 and CD81 were detected in both Exo-normoxic and Exo-hypoxic, however, the levels of CD63 and CD81 were much higher in Exo-hypoxic than conditions for both cell lines as shown in Fig. 1b. In addition, higher concentrations of CDEs were observed in the Exo-hypoxic conditions in both SW480 and HCT116 cells (Fig. 1c). These data indicated that hypoxic 


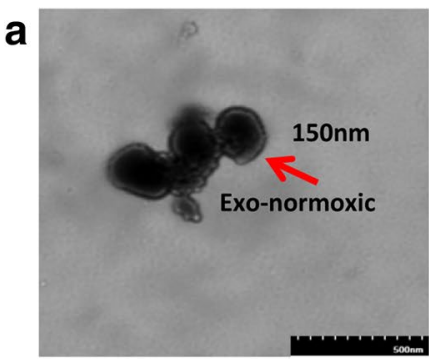

Exo-normoxic

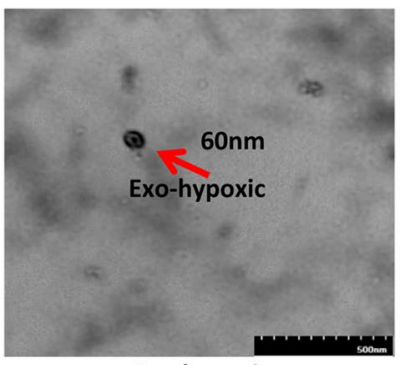

Exo-hypoxic

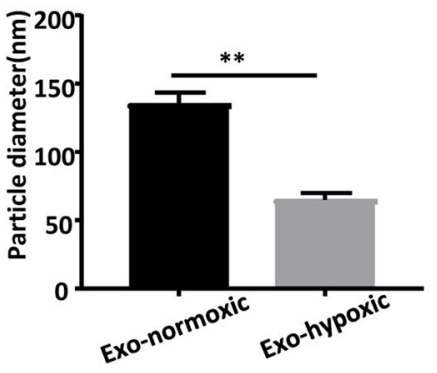

C

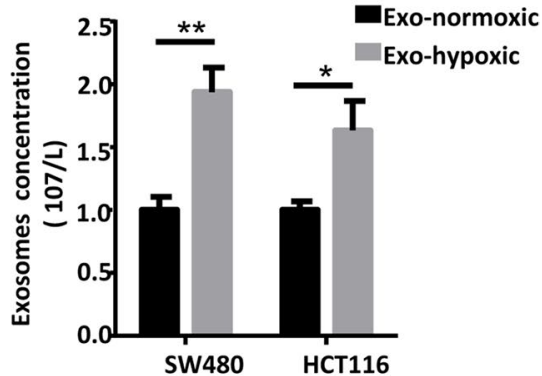

b

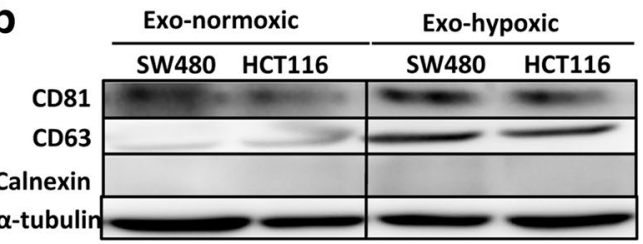

d DAPI

PKH67
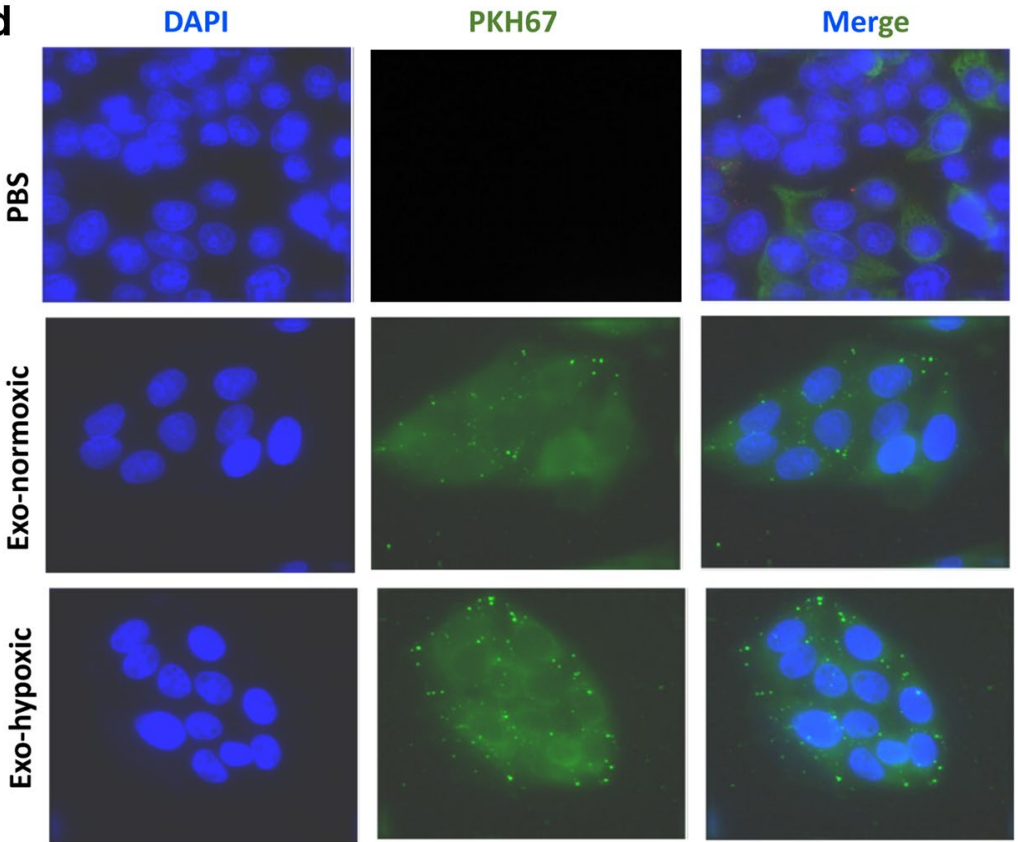

Fig. 1 The secretion of CDEs is increased when colon cancer cells were exposed to a hypoxia environment. a Exo-normoxic and Exo-hypoxic were analyzed by electron microscopy and representative photomicrographs are shown (arrow points to exosomes, magnification, $\times 200,000$, scale bar, $50 \mathrm{~nm}$. The quantification of CDEs number in the picture on the right $(\mathrm{n}=4)$. $\mathbf{b}$ Exo-normoxic and Exo-hypoxic collected through precipitation method were lysed and protein expressions of CD81, CD63, Calnexin, and a-tubulin were analyzed by Western blotting after equal amount of protein loading. c The number of exosomes released from SW480 and HCT116 cell line in normoxia and hypoxia respectively. $\mathbf{d}$ Immunofluorescence imaging analysis PKH67-labeled exosomes (Exo-hypoxic and Exo-normoxic) derived from the CRC cells were taken up by their own tumor cells $\left({ }^{*} \mathrm{P}<0.05 ;{ }^{* *} \mathrm{P}<0.01\right)$

conditions induce more but smaller exosome release from CRC cells than normoxic conditions.

To examine whether CDEs can be absorbed by CRC cells, we labeled CDEs with PKH-67 green dye and incubated these PKH67-labeled CDEs (PKH-67 are lipophilic cell tracking dyes with long aliphatic tails into lipid regions of exactly lipophilic on exosomes CDEs) with SW480 cells for $12 \mathrm{~h}$. As illustrated in Fig. 1d, CDEs from both Exo-normoxic and Exo-hypoxic conditions were significantly absorbed by SW480 cells. These observations 
suggested that exosomes derived from hypoxic CRC cells were more susceptible to be absorbed by CRC cells.

\section{Exo-hypoxic conditions promote more colon cancer cell line self-proliferation than Exo-normoxic conditions}

To examine the effects of CDEs on SW480 cell proliferation, we incubated SW480 cells with CDEs. As illustrated in Fig. 2a, incubation with Exo-normoxic or Exo-hypoxic CDEs $(10,20$ and $40 \mu \mathrm{g} / \mathrm{ml})$ for $24 \mathrm{~h}$ markedly increased the number of SW480 cells compared to PBS-treated group. Meanwhile, there was a higher number of SW480 cells with incubation of $20 \mu \mathrm{g} / \mathrm{ml}$ Exo-hypoxic than that with $20 \mu \mathrm{g} / \mathrm{ml}$ Exo-normoxic (Fig. 2a). Incubation with Exo-normoxic or Exo-hypoxic CDEs for $24 \mathrm{~h}$ also significantly increased the number of HCT116 cells compared to PBS-treated groups (Fig. 2b). There was a higher number of HCT116 cells with incubation of $10 \mu \mathrm{g} / \mathrm{ml}$ Exo-hypoxic than with $10 \mu \mathrm{g} / \mathrm{ml}$ Exo-normoxic (Fig. 2b).

We also checked the levels of cell cycle-related proteins, including cyclin D1 and Hes1. As illustrated in Fig. 2c, Exo-normoxic CDEs markedly increased cyclin D1 but not Hes1 expression in SW480 and HCT116 cells compared to PBS treatment. Additionally, incubation with Exo-hypoxic induced much higher levels of Cyclin D1 proteins in SW480 cells compared to incubation with Exo-normoxic (Fig. 2d).

\section{CDEs promote cell proliferation by reducing mitosis duration times of colon cancer cells}

To identify whether CDEs promote colon cancer cell proliferation by shortening the mitosis duration, SW480 cells were incubated with Exo-normoxic or Exo-hypoxic CDEs. During the experiment, live cell imaging was carried out. Morphological characteristics of chromosome

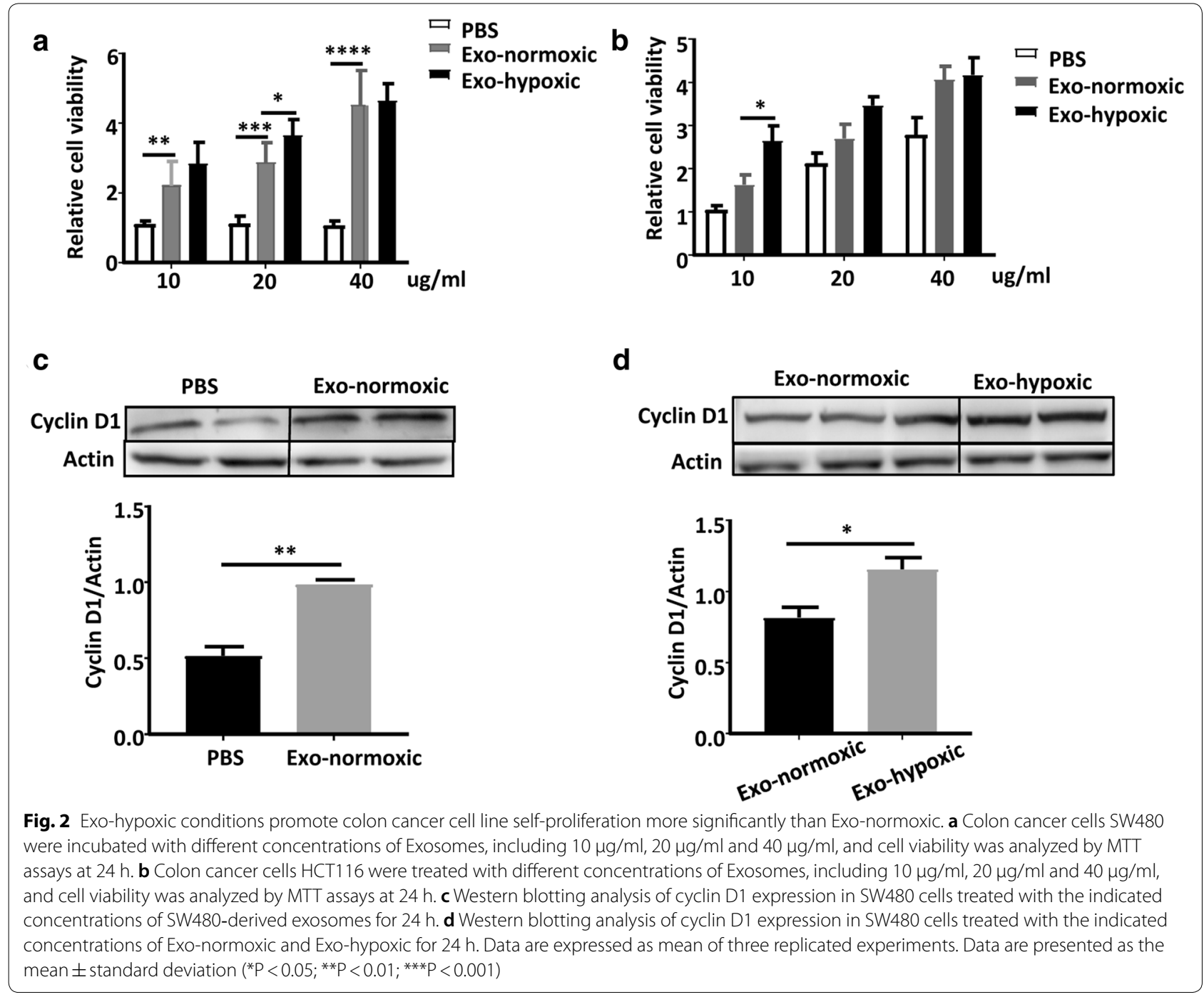


and nuclei in different period of mitosis was observed by the confocal microscope and are shown in Fig. 3a, including prophase, metaphase, telophase. Our data revealed that the expression of mitotic marker phospho-histone
$\mathrm{H} 3$ (pH3) was comparable between treatment with Exonormoxic and Exo-hypoxic CDEs. Interestingly, we observed that the changes of morphological characteristics of chromosome and nucleus in different periods of
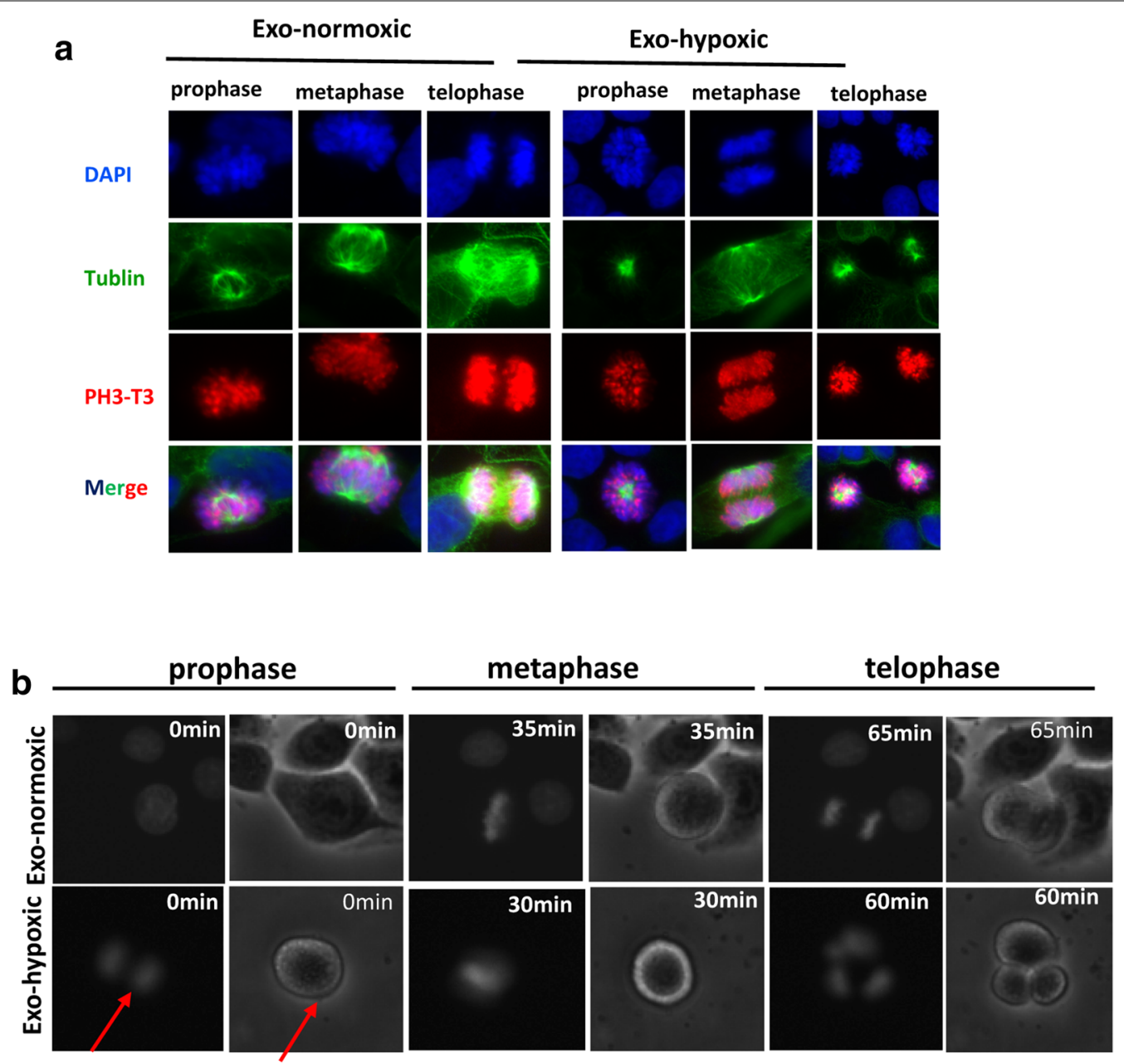

Chromosome Nucleus

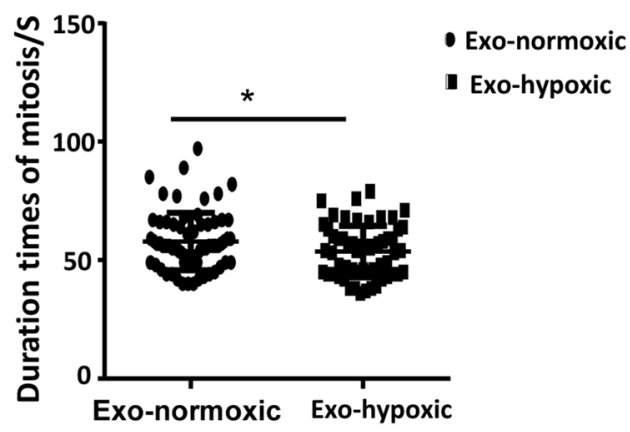

Fig. 3 CDEs promote cell proliferation by shortening mitosis duration colon cancer cells. a Representative cell morphology of different stages of mitosis $24 \mathrm{~h}$ post the treatment of Exo-normoxic and Exo-hypoxic. $\mathbf{b}$ The changes of the morphology of nucleus and chromosome of tumor cells at different stages of mitosis were measured and the statistical results of duration mitosis were obtained $\left({ }^{*} \mathrm{P}<0.05\right)$ 
mitosis were not synchronized (Fig. 3b). Exo-hypoxictreated SW480 cells started cell division earlier than Exo-normoxic-treated cells, suggesting that Exo-hypoxic promotes cell proliferation by shortening mitosis duration times of colon cancer cells (Fig. 3c).

\section{CDEs from Exo-hypoxic conditions induce higher levels of phosphorylated STAT3 in colon cancer cells than that from Exo-normoxic conditions}

As reported previously, persistent STAT3 activation in colon cancer is associated with enhanced cell proliferation and tumor growth [18], and tumor derived exosomes have been shown to activate the phosphorylation of STAT3 in ovarian cancer cells [12]. Thus, we wondered whether CDEs are able to activate STAT3 in CRC cells. As illustrated in Fig. 4a, incubation with CDEs from Exonormoxic conditions induced greater STAT3 activation in both SW480 and HCT116 cells than PBS treatment. Moreover, Exo-hypoxic treatment induced higher levels of pSTAT3 in SW480 and HCT116 cells than Exo-normoxic treatment (Fig. 4b).

\section{Discussion}

In this study, we found that the secretion of CDEs were increased when CRCs was exposed to a hypoxic environment. Interestingly, CRCs were able to absorb more Exo-hypoxic than Exo-normoxic. Both Exo-hypoxic and Exo-normoxic promoted the CRC cell proliferation with greater proliferation induced by Exo-hypoxic than by Exo-normoxic. Finally, Exo-hypoxic treatment induced stronger STAT3 activation and shorter mitosis duration in colon cancer cells than Exo-normoxic.

Hypoxia is an important feature of the tumor microenvironment. Hypoxic tumors exhibit more aggressive phenotypes and are associated with poor patient outcome in a wide variety of cancers [7]. In those hypoxic tissue regions, cancer cells remodel their unfavorable micro-environment for tumor, which usually cause resistance to cancer therapy [19]. Exosomes are vital mediators of intercellular communication that can transfer the hypoxic cells' phenotype to non-hypoxic cells through the production of exosomes $[17,20]$. Increasing studies have indicated that hypoxia promotes exosome secretion in various types of tumors [6], which may influence tumor initiation, growth and progression [7]. Multiple proteins or RNAs in cancer cells are involved in the release process of exosomes. Especially, the small GTPases, RAB27A and RAB27B, were participated in exosome secretion in various human tumor cells $[21,22]$. In addition, there are reports showing that heat shock proteins (HSP70 and HSP90) were increased in exosomes due to cellular stresses [23]. These proteins inhibit cell apoptosis and increase cell proliferation so these exosomes provide a strong stimulus to the microenvironment that can facilitate the growth of cancers. In our study, we also found that the secretion of CDEs was markedly increased when CRCs were exposed to a hypoxia environment. However, the underlying molecular mechanisms remain unknown. More studies are required to be done to elucidate the mechanisms involved in the regulation of the number

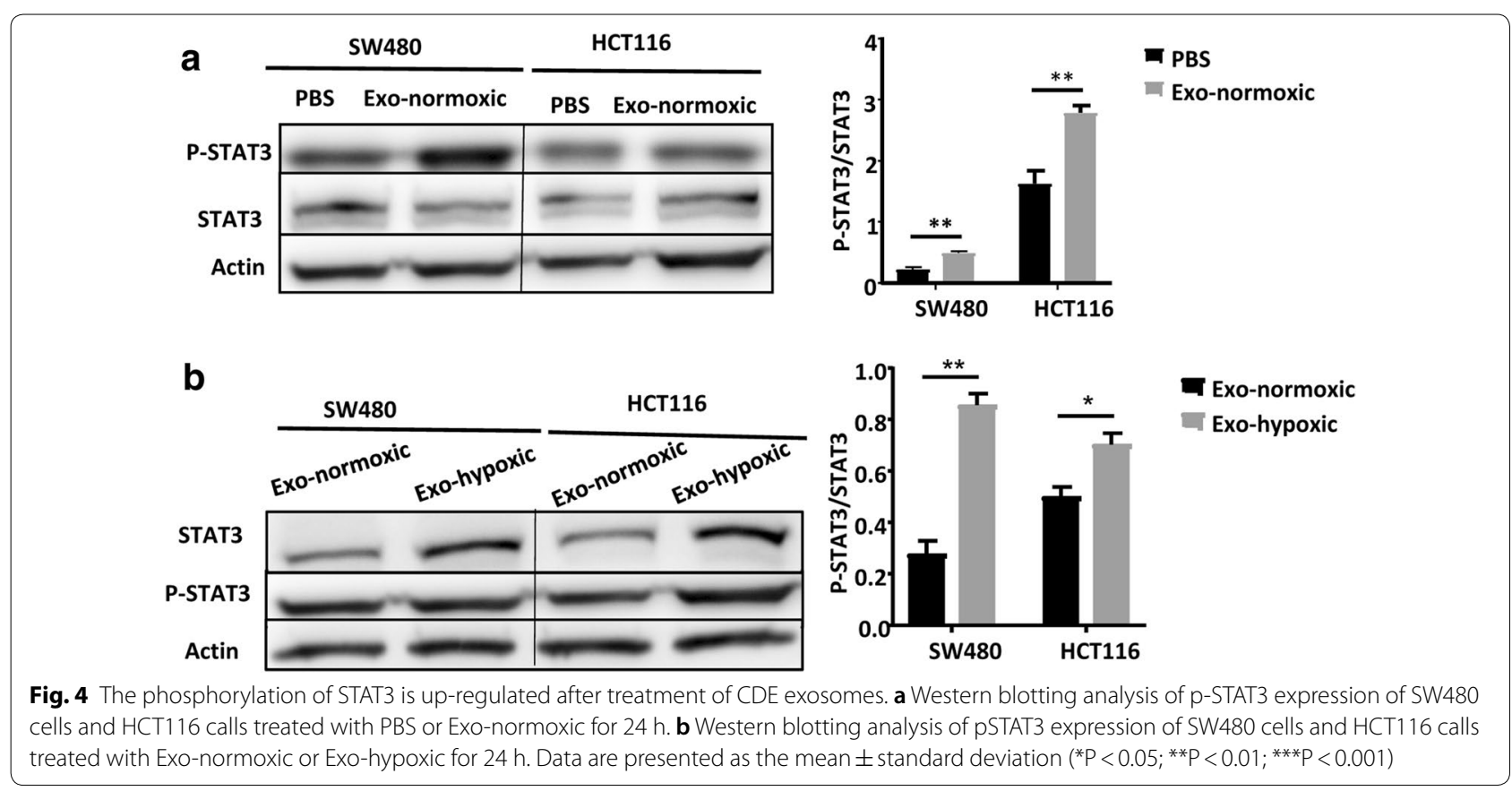


and size of exosomes secreted by CRC cells under different culture conditions.

It has been reported that hypoxia can promote the proliferation of CRCs, however, the mechanisms that hypoxia influence tumorigenesis are not fully understood. Exosomes are small microvesicles released from many types of cells and act as carriers of molecular information of cell-to-cell communication, transferring cargo content involved in many processes from parent to recipient cells. Exosomes provide the ability to transmit messages between cells at a distance and their roles in long distance communication have been well established [24]. The discovery of functional, transportable mRNA and miRNA within exosomes further increases the complexity of cellto-cell communication. They can fuse with the recipient cells and deliver their contents into the cytoplasm of the recipient cell and perturb the recipient cell, especially since miRNA can mediate RNA interference [25]. They can also carry a combination of ligands to engage several cellular receptors at once modulating changes in the recipient cell [26]. CRC may modulate their own growth via autocrine signals provided by exosomes. The promotion roles of tumor cell proliferation by exosomes from colon cancer has been widely reported. For example, autocrine signals mediated by exosomes from the non-small cell lung cancer cell lines, glioma cells and gastric cancer cell lines improve cellular proliferation by increasing phosphorylation of Akt and extracellular signal regulated kinase [27]. The promotion roles of tumor cell proliferation by exosomes from colon [28] cancer has been reported in many studies. The contents carried by exosomes (including nucleic acids and proteins) have been confirmed as the cause for enhanced proliferation of CRCs. For example, $\triangle$ Np73 mRNA is enriched in CDEs. Incubation with CDEs containing $\triangle \mathrm{Np} 73$ significantly increases the proliferative potential of target cells by inhibiting the function of tumor suppressor gene P53 $[28,29]$. CRC Wnt5b-associated exosomes promote cancer cell migration and proliferation by inducing matrix metallopeptidases (MMPs) [30]. Also, MVP-mediated exosomal sorting of miR-193a promotes colon cancer progression through targeting of Caprin1, which upregulates Ccnd2 and c-Myc [14]. Our results revealed that Exo-hypoxic and Exo-normoxic significantly promote the proliferation of CRCs with greater proliferation induced by Exo-hypoxic than by Exo-normoxic, which is probably because Exo-hypoxic induce stronger STAT3 activation and shorter mitosis duration in colon cancer cells than normoxic as demonstrated in the current studies.

Chromatin instability is a major factor in the formation of tumors and shortening the length of mitosis can accelerate tumor cell proliferation [31, 32]. In our studies, we observed the localization and morphological changes of chromosomes of CRCs in various phases of mitosis after treatment with CDEs isolated from CRCs in Exo-hypoxic and Exo-normoxic condition. However, the cell mitosis duration was significantly shorter after treatment with Exo-hypoxic than with Exo-normoxic. Given that CDEs released from tumor cells carry oncogenic proteins that can alter the signaling pathways in the surrounding recipient tumor cells or normal cells in the hypoxic microenvironment [33], these oncogenic proteins may contribute to the regulation of mitosis duration. We also observed that greater STAT3 activation was observed after treatment with CDEs isolated CRCs in Exo-hypoxic than that with Exo-normoxic conditions. Activation of STAT3 has been shown to promote proliferation of colon cancer cell lines [34, 35]. Therefore, stronger STAT3 activation likely contributes to stronger CRC proliferation induced by Exohypoxic than by Exo-normoxic. In summary, CDCs from colon cancer cells can promote proliferation of themselves by shortening mitosis duration and activating STAT3. Thus, CDCs could be a novel therapeutic target for the treatment of colon cancer.

\section{Conclusion}

In summary, increased secretion of CDEs by CRCs were observed in a hypoxic environment. The Exo-hypoxic promoted the CRC cell proliferation greater than by Exonormoxic which was associated with stronger STAT3 activation and shorter mitosis duration in CRCs.

\section{Acknowledgements \\ Not applicable.}

Authors' contributions

RR and JL performed experiments, CM analyzed data, HS edited the manuscript, HW designed experiments and wrote the manuscript. All authors read and approved the final manuscript.

Funding

This study was supported by the National Nature and Science Foundation of China (81770588 and 81522009).

Availability of data and materials

All data generated or analyzed during this study are included in this published article.

Ethics approval and consent to participate Not applicable.

Consent for publication

Not applicable.

Competing interests

The authors declare that they have no competing interests.

Received: 29 May 2019 Accepted: 1 August 2019

Published online: 06 August 2019 


\section{References}

1. Kolligs FT. Diagnostics and epidemiology of colorectal cancer. Visc Med. 2016;32(3):158-64.

2. Bray F, Ferlay J, Soerjomataram I, Siegel RL, Torre LA, Jemal A. Global cancer statistics 2018: GLOBOCAN estimates of incidence and mortality worldwide for 36 cancers in 185 countries. CA Cancer J Clin. 2018;68(6):394-424

3. Herzig D, Hardiman K, Weiser M, You N, Paquette I, Feingold DL, et al. The American society of colon and rectal surgeons clinical practice guidelines for the management of inherited polyposis syndromes. Dis Colon Rectum. 2017;60(9):881-94.

4. Gillies RJ, Verduzco D, Gatenby RA. Evolutionary dynamics of carcinogenesis and why targeted therapy does not work. Nat Rev Cancer. 2012;12(7):487-93.

5. Tkach $M$, Thery C. Communication by extracellular vesicles: where we are and where we need to go. Cell. 2016;164(6):1226-32.

6. Ji H, Greening DW, Barnes TW, Lim JW, Tauro BJ, Rai A, et al. Proteome profiling of exosomes derived from human primary and metastatic colorectal cancer cells reveal differential expression of key metastatic factors and signal transduction components. Proteomics. 2013;13(10-11):1672-86.

7. Lu X, Kang Y. Hypoxia and hypoxia-inducible factors: master regulators of metastasis. Clin Cancer Res. 2010;16(24):5928-35.

8. Manoochehri Khoshinani H, Afshar S, Najafi R. Hypoxia: a double-edged sword in cancer therapy. Cancer Invest. 2016;34(10):536-45.

9. Zheng Z, Liu L, Zhan Y, Yu S, Kang T. Adipose-derived stem cell-derived microvesicle-released miR-210 promoted proliferation, migration and invasion of endothelial cells by regulating RUNX3. Cell Cycle. 2018;17(8):1026-33.

10. Wong CC, Gilkes DM, Zhang H, Chen J, Wei H, Chaturvedi P, et al. Hypoxiainducible factor 1 is a master regulator of breast cancer metastatic niche formation. Proc Natl Acad Sci USA. 2011;108(39):16369-74.

11. Ramteke A, Ting H, Agarwal C, Mateen S, Somasagara R, Hussain A, et al. Exosomes secreted under hypoxia enhance invasiveness and stemness of prostate cancer cells by targeting adherens junction molecules. Mol Carcinog. 2015;54(7):554-65.

12. Dorayappan KDP, Wanner R, Wallbillich JJ, Saini U, Zingarelli R, Suarez AA, et al. Hypoxia-induced exosomes contribute to a more aggressive and chemoresistant ovarian cancer phenotype: a novel mechanism linking STAT3/Rab proteins. Oncogene. 2018;37(28):3806-21.

13. Kim MJ, Jung BK, Cho J, Song H, Pyo KH, Lee JM, et al. Exosomes secreted by toxoplasma gondii-infected L6 cells: their effects on host cell proliferation and cell cycle changes. Korean J Parasitol. 2016;54(2):147-54.

14. Teng Y, Ren Y, Hu X, Mu J, Samykutty A, Zhuang X, et al. MVP-mediated exosomal sorting of miR-193a promotes colon cancer progression. Nat Commun. 2017;8:14448.

15. Real JM, Ferreira LRP, Esteves GH, Koyama FC, Dias MVS, Bezerra-Neto JE, et al. Exosomes from patients with septic shock convey miRNAs related to inflammation and cell cycle regulation: new signaling pathways in sepsis? Crit Care. 2018;22(1):68.

16. Yuan O, Lin C, Wagner J, Archard JA, Deng P, Halmai J, et al. Exosomes derived from human primed mesenchymal stem cells induce mitosis and potentiate growth factor secretion. Stem Cells Dev. 2019;28(6):398-409.

17. Zonneveld MI, Keulers TGH, Rouschop KMA. Extracellular vesicles as transmitters of hypoxia tolerance in solid cancers. Cancers (Basel). 2019;11(2):154.

18. Corvinus FM, Orth C, Moriggl R, Tsareva SA, Wagner S, Pfitzner EB, et al. Persistent STAT3 activation in colon cancer is associated with enhanced cell proliferation and tumor growth. Neoplasia. 2005;7(6):545-55.
19. Rohwer N, Cramer T. Hypoxia-mediated drug resistance: novel insights on the functional interaction of HIFs and cell death pathways. Drug Resist Updat. 2011;14(3):191-201.

20. Huang Z, Feng Y. Exosomes derived from hypoxic colorectal cancer cells promote angiogenesis through Wnt4-induced beta-catenin signaling in endothelial cells. Oncol Res. 2017;25(5):651-61.

21. Peinado H, Aleckovic M, Lavotshkin S, Matei I, Costa-Silva B, MorenoBueno $G$, et al. Melanoma exosomes educate bone marrow progenitor cells toward a pro-metastatic phenotype through MET. Nat Med. 2012;18(6):883-91.

22. Ostrowski M, Carmo NB, Krumeich S, Fanget I, Raposo G, Savina A, et al. Rab27a and Rab27b control different steps of the exosome secretion pathway. Nat Cell Biol. 2010;12(1):19-30; sup pp 1-13.

23. Khan S, Jutzy JM, Aspe JR, McGregor DW, Neidigh JW, Wall NR. Survivin is released from cancer cells via exosomes. Apoptosis. 2011;16(1):1-12.

24. van Niel G, D'Angelo G, Raposo G. Shedding light on the cell biology of extracellular vesicles. Nat Rev Mol Cell Biol. 2018;19(4):213-28.

25. Thery C, Zitvogel L, Amigorena S. Exosomes: composition, biogenesis and function. Nat Rev Immunol. 2002;2(8):569-79.

26. Mathieu M, Martin-Jaular L, Lavieu G, Thery C. Specificities of secretion and uptake of exosomes and other extracellular vesicles for cell-to-cell communication. Nat Cell Biol. 2019;21(1):9-17.

27. Skog J, Wurdinger T, van Rijn S, Meijer DH, Gainche L, Sena-Esteves M, et al. Glioblastoma microvesicles transport RNA and proteins that promote tumour growth and provide diagnostic biomarkers. Nat Cell Biol. 2008;10(12):1470-6.

28. Soldevilla B, Rodriguez M, San Millan C, Garcia V, Fernandez-Perianez R, Gil-Calderon B, et al. Tumor-derived exosomes are enriched in DeltaNp73, which promotes oncogenic potential in acceptor cells and correlates with patient survival. Hum Mol Genet. 2014;23(2):467-78.

29. Soldevilla B, Diaz R, Silva J, Campos-Martin Y, Munoz C, Garcia V, et al. Prognostic impact of DeltaTAp73 isoform levels and their target genes in colon cancer patients. Clin Cancer Res. 2011;17(18):6029-39.

30. Harada T, Yamamoto H, Kishida S, Kishida M, Awada C, Takao T, et al. Wnt5b-associated exosomes promote cancer cell migration and proliferation. Cancer Sci. 2017;108(1):42-52

31. Gisselsson D, Björk J, Höglund M, Mertens F, Dal Cin P, Åkerman M, et al. Abnormal nuclear shape in solid tumors reflects mitotic instability. Am J Pathol. 2001;158(1):199-206.

32. Yang C, Shi X, Huang Y, Zhang Z, Cooke HJ, Wang M, et al. Rapid proliferation of daughter cells lacking particular chromosomes due to multipolar mitosis promotes clonal evolution in colorectal cancer cells. Cell Cycle. 2012;11(14):2650-9.

33. Hoshino A, Costa-Silva B, Shen TL, Rodrigues G, Hashimoto A, Tesic Mark $M$, et al. Tumour exosome integrins determine organotropic metastasis. Nature. 2015;527(7578):329-35.

34. Lin L, Liu A, Peng Z, Lin HJ, Li PK, Li C, et al. STAT3 is necessary for proliferation and survival in colon cancer-initiating cells. Cancer Res. 2011;71(23):7226-37.

35. Lu R, Zhang YG, Sun J. STAT3 activation in infection and infection-associated cancer. Mol Cell Endocrinol. 2017;451:80-7.

\section{Publisher's Note}

Springer Nature remains neutral with regard to jurisdictional claims in published maps and institutional affiliations. 